\title{
Non-Invasively Distinguishing Progress of Liver Fibrosis by Visualizing Hepatic Platelet Derived Growth Factor Receptor-Beta Expression with an MRI Modality in Mice
}

\section{Ling Wu}

Zhongshan Hospital Fudan University https://orcid.org/0000-0001-6574-6493

\section{Xiao-quan Huang}

Zhongshan Hospital Fudan University

$\mathrm{Na} \mathrm{Li}$

Zhongshan Hospital Fudan University

\section{Cao Xie}

Zhongshan Hospital Fudan University

\section{Sheng-xiang Rao}

Zhongshan Hospital Fudan University

\section{Shi-yao Chen}

Zhongshan Hospital Fudan University

Feng Li ( $\square$ 13661654285@163.com )

Zhongshan Hospital Fudan University

\section{Research}

Keywords: Liver fibrosis, Hepatic stellate cells, Platelet-derived growth factor receptor- $\beta$, Cyclic peptides, MRI modality

Posted Date: August 11th, 2020

DOI: https://doi.org/10.21203/rs.3.rs-53243/v1

License: (c) (i) This work is licensed under a Creative Commons Attribution 4.0 International License. Read Full License 


\section{Abstract}

Background: Activated hepatic stellate cells are the most critical cell responsible for liver fibrosis. In liver fibrogenesis, platelet-derived growth factor is the most prominent mitogen for hepatic stellate cells. This study aims to explore the potential of gadolinium (Gd)-labeled cyclic peptides (pPB) targeted to plateletderived growth factor receptor- $\beta$ (PDGFR- $\beta$ ) as a magnetic resonance imaging (MRI) radiotracer to identify the progress of liver fibrosis by imaging hepatic PDGFR- $\beta$ expression.

Results: Hepatic PDGFR- $\beta$ expression level was found to be paralleled with the severity of liver fibrosis, which was increased with the progression of fibrosis and reduced with the regression. Majority of cells expressing PDGFR- $\beta$ was determined to be activated hepatic stellate cells in fibrotic livers. Cultureactivated human hepatic stellate cells expressed abundant PDGFR- $\beta$, and FITC-labeled pPB could bind to human hepatic stellate cells in a concentration and time dependent manner. With Gd-labeled pPB as a tracer, an MRI modality demonstrated that the relative hepatic T1-weighed MR signal value was increased progressively along with severity of hepatic fibrosis and reduced with the remission.

Conclusion: Hepatic PDGFR- $\beta$ expression reflects the progress of hepatic fibrosis, and MR imaging using Gd-labeled pPB as a tracer may distinguish different stages of liver fibrosis in mice.

\section{Introduction}

Liver fibrosis is a wound-healing response to various liver injuries, such as alcohol, viruses and hepatotoxic materials, and may be protective to a certain extent for fibrosis has been found to be associated with increased resistance of hepatocytes toward these injurious stimuli. ${ }^{1}$ However, sustained or repeated liver injuries result in excessive fibrotic scars which inevitably damage hepatic lobular structure and distort hepatic vascular architecture, eventually resulting in liver dysfunction. For a long time, the prognosis of chronic liver disease (CLD) has been considered to largely depend on the severity and progression of liver fibrosis. ${ }^{2}$ More importantly, liver fibrosis is strongly associated with hepatocellular carcinoma (HCC), because the majority of human HCCs are found to occur in the setting of advanced fibrosis or cirrhosis. ${ }^{3}$ Thus, it seems imperative for the management of CLD to diagnose liver fibrosis as early as possible and stage the extent of fibrosis as accurately as possible.

Hepatic stellate cells (HSCs) are liver-specific mesenchymal cells, which are located in the space of Dissue between hepatic sinusoidal endothelial cells and hepatocytes. HSCs display two cellular phenotypes, the quiescent and activated state. In the quiescent state, HSCs contain abundant vitamin A lipid droplets; whereas in the activated state, they lose vitamin A and transdifferentiate into myofibroblast-like cells which express a-smooth muscle actin (a-SMA) and produce extracellular matrix (ECM). ${ }^{4}$ When the liver is injured due to various etiological factors, the signals from the damaged hepatocytes and immune cells provoke quiescent HSCs transdifferentiating to an activated state. In CLD where the liver injury persists or recurrent episodes, HSCs become continuous or repeated activation, 
which subsequently produce massive ECM and inevitably result in liver fibrosis and even cirrhosis. Thus, activated HSCs are generally considered to be the most critical cell responsible for liver fibrosis. ${ }^{5,6}$

In liver fibrogenesis, platelet-derived growth factor (PDGF) is acknowledged to be the most prominent mitogen for HSCs. At present, PDGF signaling network is found to be comprised of four ligands, PDGF A$D$, and transduce their signals through two transmembrane receptors, PDGFR- $\alpha$ and $-\beta{ }^{7}$ It has been shown that PDGF receptor- $\beta$ (PDGFR- $\beta$ ) is abundantly expressed on activated HSCs in vitro and in vivo, and deleting PDGFR- $\beta$ on HSCs impairs their fibrogenic potential in vivo and decreases hepatic expression of a-SMA and collagen a1. ${ }^{8,9}$

The cyclic peptide $C^{\star}$ SRNLIDC* $(p P B)$ contains arginine $(R)$ and isoleucine $(I)$ amino acids, which are the receptor-binding moieties of PDGF B-chain, and can bind specifically to PDGFR- $\beta .{ }^{10}$ After modified with PPB, carriers including human serum albumin, liposome or adenovirus carriers have been shown to have a specific affinity for activated HSCs. ${ }^{10-12}$

In this study, we investigated the association between hepatic PDGFR- $\beta$ expression level and the extent of liver fibrosis. Then after PPB was modified with gadolinium $(\mathrm{Gd})$ and used as a magnetic resonance imaging (MRI) agent, we tried to develop a molecular imaging modality to distinguish the process of liver fibrosis by visualizing hepatic PDGFR- $\beta$ expression.

\section{Materials And Methods Experimental Animals}

Male C57BL/6J mice were from the Department of Experimental Animals, Fudan University (Shanghai, China). The study was approved by Institutional Ethical Committee of Animal Experimentation, and all experiments were performed according to the governmental and international guidelines on animal experimentation.

\section{Synthesis of pPB Cyclic Peptide and its derivatives}

As we described previously, ${ }^{11}$ the linear octapeptide CSRNLIDC was synthesized by the Boc-protected solid-phase peptide synthesis method and cyclized by the linkage of two cysteine residues to gain cyclic peptide (pPB). Then pPB was incubated with FITC for 2 hours in the presence of triethylamine to gain FITC-labeled pPB cyclic peptide (pPB-FITC).

pPB was labeled with Gd through 1,4,7,10-Tetraazacyclododecane-1,4,7,10-tetraacetic acid (DOTA). Firstly, pPB-DOTA was prepared. Briefly, $20 \mathrm{mg}$ of pPB was dissolved in $4.0 \mathrm{~mL}$ PBS $(0.1 \mathrm{~mol} / \mathrm{L}, \mathrm{pH} 7.4)$. Maleimido-mono-amide-DOTA (20 mg) was dissolved in $1.0 \mathrm{~mL} \mathrm{~N}, \mathrm{~N}$-Dimethylformamide and then added into pPB solution. After stirred for 2 hours at room temperature, the mixed solution was filtered, purified and freeze-dried to gain pPB-DOTA. Next, pPB-DOTA $(60 \mathrm{mg})$ was dissolved in $20.0 \mathrm{~mL}$ pure water, and $32 \mathrm{mg}$ of $\mathrm{GdCl}_{3} \cdot 6 \mathrm{H}_{2} \mathrm{O}$ was dissolved in $4.0 \mathrm{~mL}$ pure water. Then the two kinds of solutions were mixed, 
and the mixed solution was raised to a $\mathrm{PH}$ of about 6.0 by $\mathrm{NaOH}$. After being vibrated overnight at $37^{\circ} \mathrm{C}$, the mixed solution was filtered, purified and freeze-dried to gain Gd-labeled pPB cyclic peptide (pPBDOTA-Gd).

The purity of pPB and its derivatives was examined by analytical reverse phase high performance liquid chromatography, and their molecular weight was examined by electrospray ionization mass spectrometry.

\section{Expression of PDGFR- $\beta$ on Human HSCs}

Human HSC-LX2 (from Cell Bank of Chinese Academy of Sciences, Shanghai, China) were cultured in the cell medium supplemented with $5 \%$ heat-inactivated fetal bovine serum (FBS) and $1 \%$ penicillin/streptomycin in $5 \% \mathrm{CO}_{2}$ humidified atmosphere at $37^{\circ} \mathrm{C}$. After cultured for 24 hours, most of the cells were verified to be activated by examining a-SMA expression with immunofluorescent cytochemistry.

After cultured for 24 hours, HSC-LX2 were fixed with $4 \%$ paraformaldehyde and permeabilized with PBS containing $0.1 \%$ Triton X-100 and $0.1 \mathrm{mg} / \mathrm{mL}$ RNase A when appropriate. Subsequently, HSC-LX2 were incubated with primary antibodies against PDGFR- $\beta$ and $\alpha$-SMA (both 1:200 in blocking solution, Abcam, UK) at $4{ }^{\circ} \mathrm{C}$ for overnight. After incubated with secondary antibodies (Molecular Probes, Eugene, OR) and 6-diamidino-2-phenylindole (DAPI) at room temperature for 1 hour, the slides were then photographed with a fluorescence microscope (Olympus, Japan).

\section{Binding Characteristics of pPB Cyclic Peptide to Human HSCs}

Human HSC-LX2 were incubated with the primary antibodies against a-SMA at $4{ }^{\circ} \mathrm{C}$ overnight. After the cells were incubated with Texas red-conjugated secondary antibodies (Molecular Probes), they were incubated with $1 \mu \mathrm{mol} / \mathrm{L}$ of pPB-FITC solution for 24 hours at $37^{\circ} \mathrm{C}$ in the dark. Then the cells were rinsed and stained the nuclei with DAPI. After mounted, they were observed with a fluorescence microscope.

In order to assess the binding efficiency of pPB cyclic peptide at different concentrations and different incubation durations to HSCs, human HSC-LX2 were incubated respectively with pPB-FITC solution at concentrations of $0,0.04,0.2,1,5,25$ and $125 \mu \mathrm{mol} / \mathrm{L}$ for 1 hour, or with $1 \mu \mathrm{mol} / \mathrm{L}$ solution for $0,15,30$, $60,90,120$ minutes and 24 hours at $37^{\circ} \mathrm{C}$ in the dark. After incubation, these cells were washed by centrifugation at $1100 \mathrm{rpm}$ in $4{ }^{\circ} \mathrm{C}$, fixed with $4 \%$ paraformaldehyde and immediately analyzed for the uptake rate by a FACS scan flow cytometer (FACSCalibur, USA) with CellQuest software (BD Biosciences).

\section{Mice Model of Liver Fibrosis Induced by Bile Duct Ligation $(\mathrm{BDL})$}

Liver fibrosis was induced in C57BL/6J mice by the ligation of common bile duct. ${ }^{13}$ One week or four weeks after the treatment, treated mice were used for further experiments (referred to as BDL-1W and 
BDL-4W mice). And the mice subjected to sham were used as the control group.

\section{Mice Model of Liver Fibrosis Induced by $\mathrm{CCl}_{4}$ Treatment}

Liver fibrosis was also induced in $\mathrm{C} 57 \mathrm{BL} / 6 \mathrm{~J}$ mice by carbon tetrachloride $\left(\mathrm{CCl}_{4}\right)$ treatment $\left(\mathrm{CCl}_{4}\right.$ in olive oil, 1:9 (v/v), 2 ml/kg by intraperitoneal injection twice weekly). ${ }^{14}$ Eight weeks or twelve weeks after the treatment, treated mice were used for further experiments (referred to as $\mathrm{CCl}_{4}-8 \mathrm{~W}$ and $\mathrm{CCl}_{4}-12 \mathrm{~W}$ mice). Additionally, eight $\mathrm{CCl}_{4}-8 \mathrm{~W}$ mice were randomly selected to discontinue $\mathrm{CCl}_{4}$-treatment for another 4 weeks for the spontaneous regression of fibrosis (referred to as $\mathrm{CCl}_{4}-8 \mathrm{~W}+\mathrm{S} 4 \mathrm{~W}$ mice). Mice treated with the same dosage of olive oil for 12 weeks served as the control group.

\section{Histological Analysis of Hepatic Fibrosis}

After fixed in neutralized formalin, liver sections were stained with hematoxylin and eosin or Masson. Extent of liver fibrosis was semi-quantitatively scored according to the Isake staging criteria. ${ }^{15}$ For a morphometric analysis of liver fibrosis, the Masson staining (fibrotic) areas were measured as previously described. ${ }^{16}$

In addition, serum alanine aminotransferase (ALT) levels and liver hydroxyproline content were determined using assay kits (JianCheng, Nanjing, China).

\section{Quantitative real-time PCR (qRT-PCR) analysis}

The hepatic mRNA level of PDGFR- $\beta$ was quantitated using qRT-PCR analysis as described. ${ }^{16}$ The forward primer for PDGFR- $\beta$ was AATATAAGAGGAAGAGTTG, and the reverse primer was

TATACCCAAGGATTTCTA. GAPDH was used as the control, and its forward primer was TCCCTCAAGATTGTCAGCAA, and the reverse primer was AGATCCACAACGGATACATT.

\section{Immunohistochemistry Analysis}

After washed by PBS, liver sections were performed heat mediated antigen retrieval in sodium citrate buffer ( $\mathrm{pH}$ 6.0) by using microwave. After cooled and blocked with $10 \%$ serum for 1 hour, the sections were incubated with rabbit anti-mouse PDGFR- $\beta$ antibody (1:100 in blocking solution, Millipore, Massachusetts, USA) overnight at $4{ }^{\circ} \mathrm{C}$. A Biotin conjugated goat polyclonal anti-rabbit lgG (1:100 in blocking solution, Abcam) was used as secondary antibody. Ten fields (200x) from each section were randomly selected and recorded. The PDGFR- $\beta$ positive-staining areas were respectively quantified with NIN Image 1.62 software, and the percentage of positive-staining areas in each field was calculated.

\section{Immunofluorescent Co-localization of PDGFR- $\beta$ in Various Cells of Fibrotic Livers}

Immunofluorescent staining was performed to reveal the co-localization of PDGFR- $\beta$ with a-SMA (activated HSCs), CD31 (vascular endothelial cells), CD68 (macrophages) and CD163 (Kupffer cells) in the liver sections as previously described. ${ }^{16}$ Primary antibodies against PDGFR- $\beta$ (1:100; Abcam), 
monoclonal anti-SMA (1:200; Abcam), monoclonal anti-CD31 (1:100; Abcam), monoclonal anti-CD68 (1:00; Abcam) and monoclonal anti-CD163 (1:00; Abcam) were used. Secondary antibodies included Alexa Fluor 594-conjugated IgG (1:200, Jackson, Pennsylvania, USA) and Alexa Fluor 488-conjugated IgG (1:200, Jackson). Multicolored fluorescent staining of liver sections was analyzed, and the percentage of the merged yellow color region to the total PDGFR- $\beta$-stained green region in each section was calculated. Ten randomly selected amplifying fields (400x) in each section were assessed.

\section{In Vivo MRI Studies}

MR imaging was performed by using a Biospec 70/20 MRI scanner (7.0 T) to assess the accumulation of pPB-DOTA-Gd in livers after the control mice and $\mathrm{CCl}_{4}$-treated mice ( $\mathrm{n}=3$ per group) were injected intravenously with pPB-DOTA-Gd at a dose of $0.05 \mathrm{mmol} / \mathrm{kg}\left[\mathrm{Gd}^{3+}\right]$ in a total of $0.25 \mathrm{~mL}$ PBS solution, as previously described. ${ }^{17}$ Briefly, dynamic T1-weighed MR images of the liver were collected prior to and 30, 60, 90 and 120minutes after pPB-DOTA-Gd was injection. Coronal section images of the liver were acquired with a fast low-angle shot (FLASH) sequence. Hepatic signal intensity (T1 (liver)) and muscle signal intensity ( $\mathrm{T} 1$ (muscle)) were measured from the region of interest and the relative hepatic signal intensity was denoted as T1 (liver) / T1 (muscle).

\section{Statistical Analysis}

Data were presented as the mean \pm standard deviation (SD) and analyzed by a one-way analysis of variance followed with least significant difference (LSD) test. SPSS 16.0 statistical software (Chicago, USA) was used. A $p$ value less than 0.05 was considered statistically significant.

\section{Results}

\section{Expression of PDGFR- $\beta$ in Fibrotic Livers Induced by BDL}

After mice were treated with BDL for 1 weeks (BDL-1W), marked cholestasis and neutrophil infiltration were observed in hepatic lobules, and serum ALT level was significantly increased compared to the control mice (Fig. 1A and 1B). And after mice were treated with BDL for 4 weeks (BDL-4W), the normal architecture of hepatic lobule was damaged, and visible fibrotic septa and extensive bridging fibrosis were observed (Fig. 1A). Compared to the control mice, the Masson staining (fibrotic) area and the hydroxyproline content in liver tissue were both increased in BDL-treated mice, which was the highest in BDL-4W mice (Fig. 1C and 1D). These results indicated that liver fibrosis developed and progressed after mice were treated with BDL.

As shown in Fig. 1E, hepatic mRNA level of PDGFR- $\beta$ was both significantly higher in BDL-treated mice than in the control mice, which was the highest in BDL-4W mice. After hepatic slices were stained by immunohistochemistry, PDGFR- $\beta$ positive-staining area was hardly visible in the control mice. But as liver fibrosis developed and progressed, PDGFR- $\beta$ was found to be massively expressed in livers of BDL- 
treated mice, especially in BDL-4W mice (Fig. 1F). The results indicated that hepatic PDGFR- $\beta$ expression was significantly increased with the development and progression of liver fibrosis induced by BDL.

\section{Expression of PDGFR- $\beta$ in Fibrotic Livers Induced by $\mathrm{CCl}_{4}^{-}$ treatment}

Liver fibrosis was also induced in mice by $\mathrm{CCl}_{4}$-treatment in order to further investigate hepatic PDGFR- $\beta$ expression in mice with the progression and regression of liver fibrosis. After mice were treated with $\mathrm{CCl}_{4}$ for 8 weeks $\left(\mathrm{CCl}_{4}-8 \mathrm{~W}\right)$, massive hepatocytes balloon-like degenerative change and extensive fibrotic septa were observed. When $\mathrm{CCl}_{4}$-treatment was extended to 12 weeks $\left(\mathrm{CCl}_{4}-12 \mathrm{~W}\right)$, hepatic fibrosis was markedly aggravated and hepatic lobular architecture was observed to be extensively damaged. But when $\mathrm{CCl}_{4}$-treatment was withdrawn for 4 weeks after $\mathrm{CCl}_{4}$ intoxication for 8 weeks $\left(\mathrm{CCl}_{4}-8 \mathrm{~W}+\mathrm{S} 4 \mathrm{~W}\right)$, hepatocytes balloon-like degenerative changes significantly regressed and fibrotic septa markedly resolved (Fig. 2A). Compared to the control mice, serum ALT level, the Masson staining area and the hydroxyproline content in liver tissue were all significantly increased in $\mathrm{CCl}_{4}$-treated mice. However, when $\mathrm{CCl}_{4}$-treatment was withdrawn for 4 weeks, these indexes were all markedly reduced (Fig. 2B-D), which indicated that liver fibrosis significantly regressed after $\mathrm{CCl}_{4}$-treatment was withdrawn.

Compared to the control mice, hepatic mRNA level of PDGFR- $\beta$ was significantly increased in $\mathrm{CCl}_{4^{-}}$ treatment mice, which was the highest in $\mathrm{CCl}_{4}-12 \mathrm{~W}$ mice, but was markedly reduced in $\mathrm{CCl}_{4}-8 \mathrm{~W}+\mathrm{S} 4 \mathrm{~W}$ mice (Fig. 2E). As shown in Fig. 2F, after immunohistochemistry staining, extensive PDGFR- $\beta$ positivestaining area was observed in livers of $\mathrm{CCl}_{4}$-treatment mice, especially in $\mathrm{CCl}_{4}-12 \mathrm{~W}$ mice. However, there was far fewer PDGFR- $\beta$ positive-staining area in livers of $\mathrm{CCl}_{4}-8 \mathrm{~W}+\mathrm{S} 4 \mathrm{~W}$ mice. These results indicated that hepatic PDGFR- $\beta$ expression is increased with the development and progression of liver fibrosis, and reduced with the regression of fibrosis.

Additionally, no matter in BDL mice or in $\mathrm{CCl}_{4}$-treated mice, the PDGFR- $\beta$ positive-staining area was observed to be mostly located in the fibrotic septa (Fig. 1F and Fig. 2F).

\section{Localization of PDGFR- $\beta$ in Fibrotic Livers}

Liver fibrosis induced by $\mathrm{BDL}$ or $\mathrm{CCl}_{4}$-treatment was staged according to the Ishake staging criteria, and divided into two groups: mild fibrosis (Ishake stage 1-3) and advanced fibrosis (Ishake stage 4-6). To identify the cardinal cells expressing PDGFR- $\beta$ in fibrotic livers, double immunofluorescent staining was performed to image the co-localization of PDGFR- $\beta$ with a-SMA, CD31, CD68 and CD163. In the livers with advanced fibrosis, the positive staining of PDGFR- $\beta$ was shown to be largely overlapped with a-SMA staining, and slightly overlapped with CD31 staining, but scarcely overlapped with CD68 or CD163 staining (Fig. 3). And the percentage of overlapped area (yellow) of PDGFR- $\beta$ staining (red) with a-SMA positive-staining (green) was $55.14 \pm 6.37 \%$, which was markedly higher than the overlapped staining with CD31 (19.60 $\pm 4.48 \%), \operatorname{CD} 68(8.97 \pm 1.85 \%)$ and CD163 $(6.28 \pm 1.64 \%)(p<0.05$ for all comparisons). In the livers with mild fibrosis, the positive staining of PDGFR- $\beta$ was observed to be almost entirely 
overlapped with a-SMA staining, and hardly overlapped with CD31, CD68 or CD163 staining (data not shown).

Because a-SMA are thought to be the marker of activated HSCs, the cardinal cells expressing PDGFR- $\beta$ in fibrotic livers are considered to be activated HSCs.

\section{Expression of PDGFR- $\beta$ in Activated HSCs in vitro}

Double immunofluorescent staining was performed to investigate the expression of PDGFR- $\beta$ on human activated HSC in vitro. After being sub-cultured for 24 hours, HSC-LX2 transformed into an activated cell type which was positive for a-SMA staining. Most of these culture-activated HSCs were observed to be positive for PDGFR- $\beta$ staining (Fig. 4A). The result demonstrated that PDGFR- $\beta$ was abundantly expressed on activated human HSC in liver fibrogenesis.

\section{Binding Characteristics of pPB Cyclic Peptide to Activated HSCs}

pPB cyclic peptides were prepared and labeled with FITC (pPB-FITC). The purity of pPB and its derivative was above $95 \%$. The molecular weight was 873 for pPB and 1300 for pPB-FITC.

At first, the binding of pPB-FITC to HSC-LX2 was determined by confocal microscopic imaging. The fluorescent signal (green) was clearly visible in the culture-activated HSC-LX2 after incubated with $1 \mu \mathrm{mol} / \mathrm{L}$ of pPB-FITC for 24 hours (Fig. 4B), which indicated that pPB-FITC bound to activated HSCs.

Second, when activated HSC-LX2 were incubated with PPB-FITC in a series of increasing concentration from $0.04 \mu \mathrm{mol} / \mathrm{L}$ to $125 \mu \mathrm{mol} / \mathrm{L}$ for 1 hour, their fluorescence intensity was accordingly increased to approximately 6.0 to 500.0 -fold (Fig. 4C). Additionally, when activated HSC-LX2 were incubated with $1 \mu \mathrm{mol} / \mathrm{L}$ of pPB-FITC for 15 minutes to 24 hours, an approximately 9.0 to 60.0 -fold increase in their fluorescence intensity was noted accordingly (Fig. 4D). These results indicated that the binding of pPBFITC to activated human HSCs was concentration-dependent and time-dependent.

\section{Imaging the Progress of Liver Fibrosis by MRI}

pPB cyclic peptides were labeled with Gd through DOTA (pPB-DOTA-Gd) and its molecular weight was 1554.

A dynamic T1-weighed MR imaging approach was used to assess the deposition of pPB-DOTA-Gd in the livers after the radiotracers were intravenously injected in the control and $\mathrm{CCl}_{4}$-treated mice. In $\mathrm{CCl}_{4}-12 \mathrm{~W}$ mice, the relative liver T1-MR signal intensity was gradually intensified until 60 minutes post-injection of pPB-DOTA-Gd, and then gradually reduced (Fig. 5A). There was no obvious change of hepatic signal intensity in the control mice and $\mathrm{CCl}_{4}-8 \mathrm{~W}+\mathrm{S} 4 \mathrm{~W}$ mice prior to and 60 minutes after pPB-DOTA-Gd was injected; whereas hepatic signal intensity was markedly intensified in $\mathrm{CCl}_{4}-12 \mathrm{~W}$ mice and moderately intensified in $\mathrm{CCl}_{4}-8 \mathrm{~W}$ mice at 60 minutes post-injection of pPB-DOTA-Gd (Fig. 5B). Compared to the control mice, the relative liver T1-MR signal intensity was significantly increased in $\mathrm{CCl}_{4}$-treated mice, 
which was the highest in $\mathrm{CCl}_{4}-12 \mathrm{~W}$ mice (Fig. 5C). However, there was no significantly difference in the relative liver T1-MR signal intensity between the control mice and $\mathrm{CCl}_{4}$-treatment withdrawing mice. These results indicated that hepatic deposition amount of pPB-DOTA-Gd 60 minutes after injection was gradually increased in parallel with the development and progression of liver fibrosis, and markedly reduced with the regression of fibrosis. The correlation was further assessed between the extent of liver fibrosis and the relative liver T1-MR signal intensity 60 minutes after pPB-DOTA-Gd were injected. The relative liver T1-MR signal intensity showed a strong positive correlation with Ishake stage $(r=0.858, p<$ 0.01 , Fig. 5D) and Masson-staining area ( $r=0.878, p<0.01$, Fig. $5 E)$.

\section{Discussion}

At present, liver fibrosis can be assessed by invasive biopsy and non-invasive approaches including imaging techniques based on measuring liver stiffness (such as transient elastography) and serum markers. ${ }^{18}$ Liver biopsy is still widely acknowledged as the gold standard for the diagnosis and stage of liver fibrosis, but it is an invasive procedure with a risk of rare but potentially life-threatening complications, which limit its use in the long-time follow-up and wide-scale screening of CLD. ${ }^{19}$ Over the past decade, there has been tremendous advance in the development of non-invasive approaches to assess liver fibrosis. Most of these non-invasive approaches show good work at identifying the extremes of fibrosis but is undesirable in accurately differentiating intermediate stages. ${ }^{18-20}$

In addition, liver fibrosis is a dynamic process, and the total amount of fibrous tissue depends on the synthesis, deposition, accumulation and degradation of ECM. ${ }^{21}$ More importantly, in the past decades, it has been convincingly demonstrated that after the etiologies are successfully removed, the fibrotic liver can revert to a less fibrotic or even normal architecture in both rodent model of liver fibrosis and patients with CLD, especially at the early stage of fibrosis. ${ }^{22,23}$ Hence, it may be more rational for the management of CLD to identify liver fibrosis at an advance stage or at a remission stage than to diagnose and stage fibrosis simply, which is crucial for clinicians to decide whether to take more aggressive measures to deal with CLD. So far, however, neither liver biopsy nor the existing non-invasive approaches can accurately predict the progress of liver fibrosis.

The signal transduced by PDGFR- $\beta$ is acknowledged to be the most potent among the mitogenic pathways in HSCs. Kocabayoglu $\mathrm{P}$ et al. found that hepatic fibrosis accumulation was increase at least in part through increased HSC numbers by PDGFR- $\beta$ activation and the lack of PDGFR- $\beta$ on primary HSCs led to decreased expression of collagen I. ${ }^{9}$ In this study, both in $\mathrm{BDL}$-induced and in $\mathrm{CCl}_{4}$-induced mouse model of liver fibrosis, hepatic expression level of PDGFR- $\beta$ was found to be significantly increased with the development and progression of fibrosis. The two kinds of mouse model with liver fibrosis mimic different pathogenesis of fibrosis, which is induced by cholestasis and the other is by toxin-induced hepatocellular injury. More importantly, when $\mathrm{CCl}_{4}$-treatment was withdrawn for 4 weeks after $\mathrm{CCl}_{4}$ intoxication and liver fibrosis was found to be obviously regressed, hepatic expression level of PDGFR- $\beta$ 
was observed to be markedly reduced. Thus, hepatic PDGFR- $\beta$ expression was verified to be parallelly associated with the process of liver fibrosis.

Activated HSCs are the major ECM-producing cells in the liver, and it seems crucial for predicting the prognosis of CLD to distinguish the continuous or repeated activated state of HSCs. To date, there is still no non-invasive approach to distinguish the status of HSCs, and pathological examination is the only mean to identify the status of HSCs. In the study, the positive staining of PDGFR- $\beta$ was observed to be mainly overlapped with a-SMA staining no matter in livers with mild fibrosis or with advanced fibrosis, so activated HSCs were the cardinal cells expressing PDGFR- $\beta$ in fibrotic livers. In vitro, culture-activated human HSCs (LX2) were also observed to express abundant PDGFR- $\beta$. Thus, hepatic expression level of PDGFR- $\beta$ was strongly associated with the number of activated HSCs. The continuous or repeated activation of HSCs leads to the development and progression of liver fibrosis. ${ }^{21,} 22$ Additionally, for the reversibility of liver fibrosis, it has been demonstrated to be critical to reduce the number of activated HSCs by apoptosis and deactivation. ${ }^{22-25}$ Hence, it was feasible to identify the progression or regression of liver fibrosis by reflecting the number of activated HSCs with visualizing hepatic expression level of PDGFR- $\beta$.

pPB cyclic peptides can bind specifically to PDGFR- $\beta$ and show a well targeting property to activated HSCs. In our previous study, the surface modification with pPB was found to enhance the target effect of interferon-y liposomes to activated HSCs, and increase the anti-fibrotic effect of interferon-y. ${ }^{11}$ In the present study, PPB labeled with FITC was observed to bind to activated HSC-LX2 and the binding was in a concentration-dependent and time-dependent manner. In this study, MRI was used to image the livers for it is acknowledged to be the highest spatial resolution to soft tissues among the existing imaging modalities in clinic. After pPB was labeled with Gd and used as an MRI tracer, mice with different extent of liver fibrosis showed significantly differences in the relative liver T1-MR signal intensity, which was increased with the development and progression of fibrosis and reduced with the regression of fibrosis. Thus, the molecular imagining approach can be helpful to evaluate whether CLD would continue to aggravate or get improvement after certain treatments by predicting the progression or regression of fibrosis.

\section{Conclusion}

In the present study, hepatic expression level of PDGFR- $\beta$ was observed to be closely related with the progress of liver fibrosis, which was significantly increased with the development and progression of fibrosis and reduced with the regression of fibrosis. Activated HSCs were found to be the cardinal cell expressing PDGFR- $\beta$ in fibrotic livers. After pPB cyclic peptides were labeled with Gd and used as an MRI tracer, the progress of liver fibrosis was identified by visualizing hepatic PDGFR- $\beta$ expression with an MR imaging modality.

\section{Abbreviations}


CLD, chronic liver diseases; HCC, hepatocellular carcinoma; HSCs, hepatic stellate cells; a-SMA, a-smooth muscle actin; ECM, extracellular matrix; PDGF, platelet-derived growth factor; PDGFR- $\beta$, PDGF receptor- $\beta$; pPB, cyclic peptide C*SRNLIDC*; Gd, gadolinium; MRI, magnetic resonance imaging; pPB-FITC, FITClabeled pPB cyclic peptide; DOTA, 1,4,7,10-Tetraazacyclododecane-1,4,7,10-tetraacetic acid; pPB-DOTAGd, Gd-labeled pPB cyclic peptide; FBS, fetal bovine serum; DAPI, 6-diamidino-2-phenylindole; BDL, bile duct ligation; $\mathrm{CCl}_{4}$, carbon tetrachloride; $\mathrm{ALT}$, alanine aminotransferase.

\section{Declarations}

\section{Ethics approval and consent to participate}

All animal procedures were performed under the guidelines of the institutional review board and the ethics committee of Zhongshan Hospital, Fudan University.

\section{Consent for publication}

All the authors have approved the manuscript and agree with submission to your esteemed journal.

\section{Availability of data and materials}

All data generated or analyzed during this study are included in this published article.

\section{Competing interests}

The authors declare that they have no competing interests.

\section{Funding}

This work was supported by the National Natural Science Foundation of China (Grant NO. 81270007 \& 81670513 to Li F), Shanghai Talent Development Funds (Grant NO. 201304 to Li F), Shanghai Rising-Star Program (Grant NO. 13QA1400700 to Li F), And Shanghai Outstanding Young Talent Training Plan in Health System (Grant NO. 13 YO56 to Li F).

\section{Authors' contributions}

FL, LW and XQH conceived the idea and designed the experiments. LW and XQH conducted the majority of the experiments under the help of NL, CX and SXR. FL, LW and XQH wrote the manuscript after collecting and analyzing data together with $\mathrm{NL}, \mathrm{CX}$ and SXR. SYC and FL provided help in animal experiments and modifying the manuscript. All authors read and approved the final manuscript

\section{Acknowledgements}

Not applicable. 


\section{References}

1. Bourbonnais E, Raymond VA, Ethier C, Nguyen BN, El-Leil MS, Meloche S, et al. Liver fibrosis protects mice from acute hepatocellular injury. Gastroenterology 2012;142:130-9.

2. Castera L, Pinzani M. Non-invasive assessment of liver fibrosis: are we ready? Lancet 2010;375:1419-20.

3. Seitz HK, Stickel F. Risk factors and mechanisms of hepatocarcinogenesis with special emphasis on alcohol and oxidative stress. Biol Chem 2006;387:349-60.

4. Yin C, Evason KJ, Asahina K, Stainier DY. Hepatic stellate cells in liver development, regeneration, and cancer. J Clin Invest 2013;123:1902-10.

5. Schuppan D, Kim YO. Evolving therapies for liver fibrosis. J Clin Invest 2013;123:1887-901.

6. Trautwein C, Friedman SL, Schuppan D, Pinzani M. Hepatic fibrosis: Concept to treatment. J Hepatol 2015;62 Suppl 1:S15-24.

7. Pinzani M. PDGF and signal transduction in hepatic stellate cells. Front Biosci 2002;7:d1720-6.

8. Wong L, Yamasaki G, Johnson RJ, Friedman SL. Induction of beta-platelet-derived growth factor receptor in rat hepatic lipocytes during cellular activation in vivo and in culture. J Clin Invest 1994;94:1563-9.

9. Kocabayoglu P, Lade A, Lee YA, Dragomir AC, Sun X, Fiel MI, et al. $\beta$-PDGF receptor expressed by hepatic stellate cells regulates fibrosis in murine liver injury, but not carcinogenesis. $J$ Hepatol 2015;63:141-7.

10. Beljaars L, Weert B, Geerts A, Meijer DK, Poelstra K. The preferential homing of a platelet derived growth factor receptor-recognizing macromolecule to fibroblast-like cells in fibrotic tissue. Biochem Pharmacol 2003;66:1307-17.

11. Li F, Li QH, Wang JY, Zhan CY, Xie C, Lu WY. Effects of interferon-gamma liposomes targeted to platelet-derived growth factor receptor-beta on hepatic fibrosis in rats. J Control Release 2012;159:261-70.

12. Schoemaker MH, Rots MG, Beljaars L, Ypma AY, Jansen PL, Poelstra K, et al. PDGF-receptor betatargeted adenovirus redirects gene transfer from hepatocytes to activated stellate cells. Mol Pharm 2008;5:399-406.

13. Meng F, Onori P, Hargrove L, Han Y, Kennedy L, Graf A, Hodges K, et al. Regulation of the histamine/VEGF axis by miR-125b during cholestatic liver injury in mice. Am J Pathol 2014;184:66273.

14. Jeong WI, Park O, Radaeva S, Gao B. STAT1 inhibits liver fibrosis in mice by inhibiting stellate cell proliferation and stimulating NK cell cytotoxicity. Hepatology 2006;44:1441-51.

15. Ishak K, Baptista A, Bianchi L, Callea F, De Groote J, Gudat F, et al. Histological grading and staging of chronic hepatitis. J Hepatol 1995;22:696-9.

16. Li F, Song Z, Li Q, Wu J, Wang J, Xie C, et al, Molecular imaging of hepatic stellate cell activity by visualization of hepatic integrin av $\beta 3$ expression with SPECT in rat. Hepatology 2011;54:1020-30. 
17. Li F, Yan H, Wang J, Li C, Wu J, Wu S, et al. Non-invasively differentiating extent of liver fibrosis by visualizing hepatic integrin av $\beta 3$ expression with an MRI modality in Mice. Biomaterials 2016;102:162-74.

18. Hagan M, Asrani SK, Talwalkar J. Non-invasive assessment of liver fibrosis and prognosis. Expert Rev Gastroenterol Hepatol 2015;9:1251-60.

19. Castera L, Bedossa P. How to assess liver fibrosis in chronic hepatitis C: serum markers or transient elastography vs. liver biopsy? Liver Int 2011;31 Suppl 1:13-7.

20. Singh S, Muir AJ, Dieterich DT, Falck-Ytter YT. American Gastroenterological Association Institute Technical Review on the Role of Elastography in Chronic Liver Diseases. Gastroenterology 2017;152:1544-77.

21. Friedman SL. Liver fibrosis-from bench to bedside. J Hepatol 2003;38 Suppl 1:S38-53.

22. Lee YA, Wallace MC, Friedman SL. Pathobiology of liver fibrosis: a translational success story. Gut 2015;64:830-41.

23. Troeger JS, Mederacke I, Gwak GY, Dapito DH, Mu X, Hsu CC, et al. Deactivation of hepatic stellate cells during liver fibrosis resolution in mice. Gastroenterology 2012;143:1073-83.

24. Iredale JP, Benyon RC, Pickering J, et al. Mechanisms of spontaneous resolution of rat liver fibrosis. Hepatic stellate cell apoptosis and reduced hepatic expression of metalloproteinase inhibitors. J Clin Invest 1998;102:538-49.

\section{Figures}

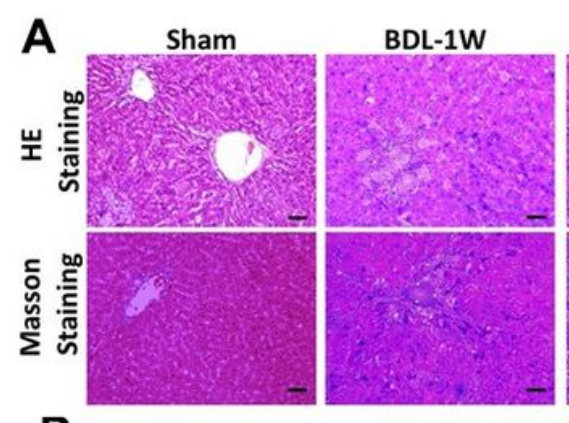

D

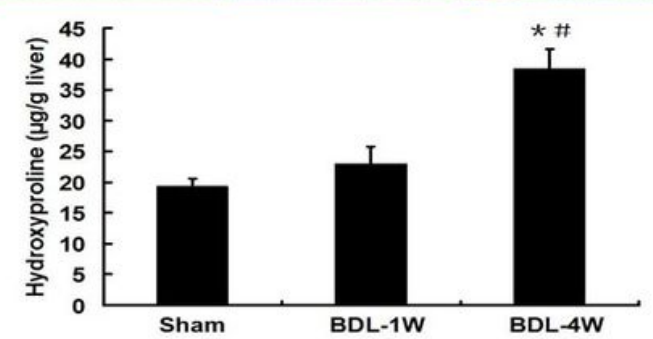

E

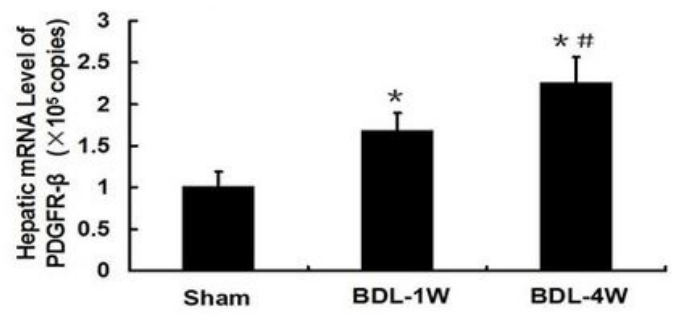

BDL-4W
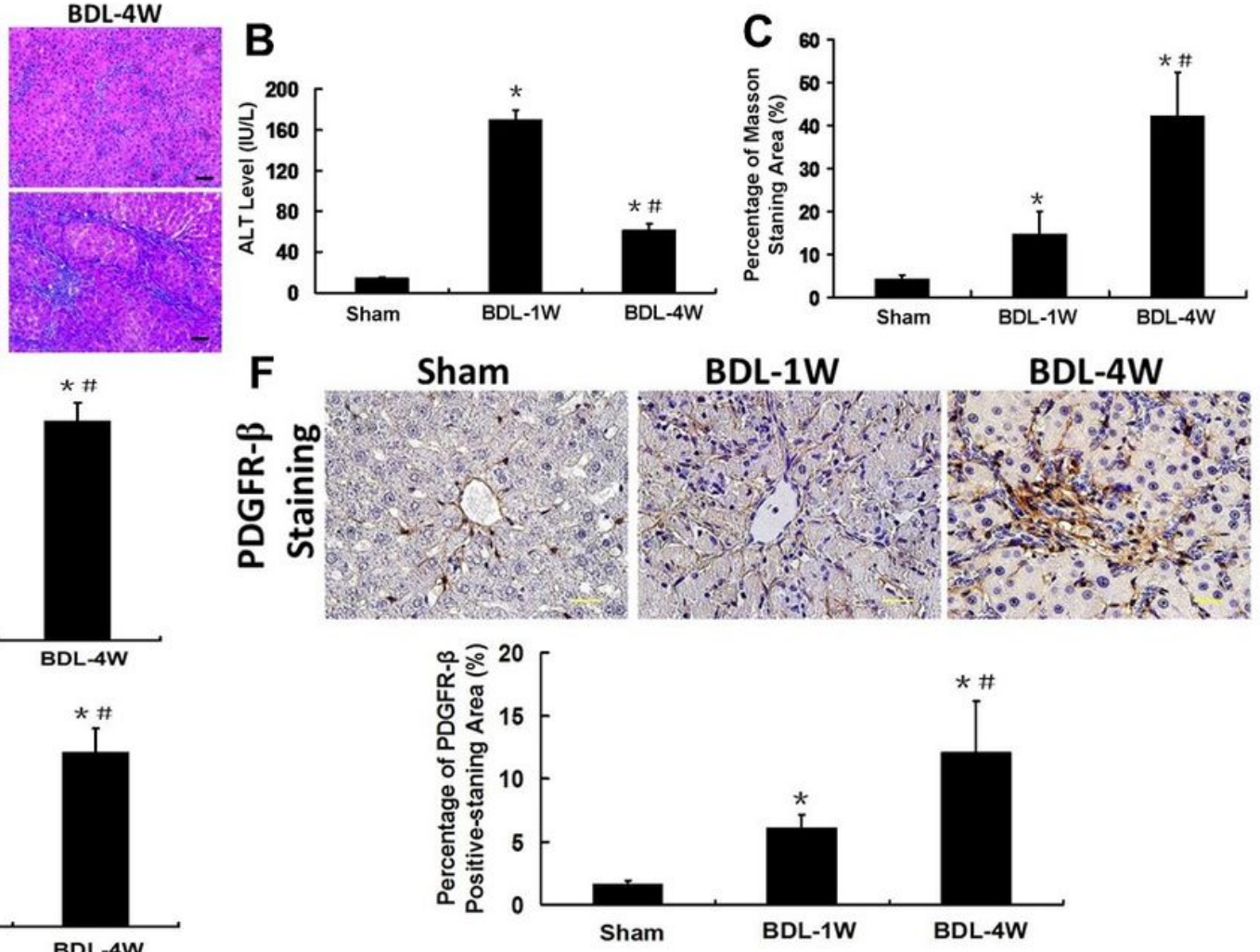


\section{Figure 1}

Expression of PDGFR- $\beta$ in fibrotic livers treated with bile duct ligation (BDL). Liver fibrosis was induced in mice by BDL for 1 and 4 weeks (referred to as BDL-1W and BDL-4W). The mice subjected to sham served as a control group. ( $\mathrm{n}=8$ per group) (A) Representative micrographs of hepatic histology stained with H\&E $(200 x)$ and Masson (100x). Scale bars $=100 \mu \mathrm{m}$. (B) Comparison of serum alanine aminotransferase (ALT) level in BDL-treated and control groups. (C) Comparison of the Masson staining (fibrotic) area in BDL-treated and control groups. For semi-quantitative analysis of liver fibrosis, 10 fields (100x) from each section were randomly selected, recorded and the Masson staining areas were measured. (D) Comparison of hydroxyproline content in liver tissue in BDL-treated and control groups. Hydroxyproline content in liver tissue was determined spectrophotometrically and expressed by $\mu \mathrm{g} / \mathrm{g}$ liver tissue. (E) Comparison of hepatic PDGFR- $\beta$ mRNA level in BDL-treated and control groups. Hepatic mRNA levels of PDGFR- $\beta$ examined by quantitative real-time RT-PCR analysis. Data are expressed in means \pm SD. (F) Representative micrographs of hepatic PDGFR- $\beta$ expression stained by immunohistochemistry analysis $(200 \times$, Scale bars $=100 \mu \mathrm{m})$. For semi-quantitative analysis of hepatic PDGFR- $\beta$ expression level, 10 fields from each section were randomly selected, recorded and the PDGFR- $\beta$ positive-staining (brown) area was measured. Then the percentage of the PDGFR- $\beta$ positive-staining area was compared in BDLtreated and control groups. In all panels, $\llbracket p<0.05$ versus sham group; $₫ p<0.05$ versus $B D L-1 W$ group.

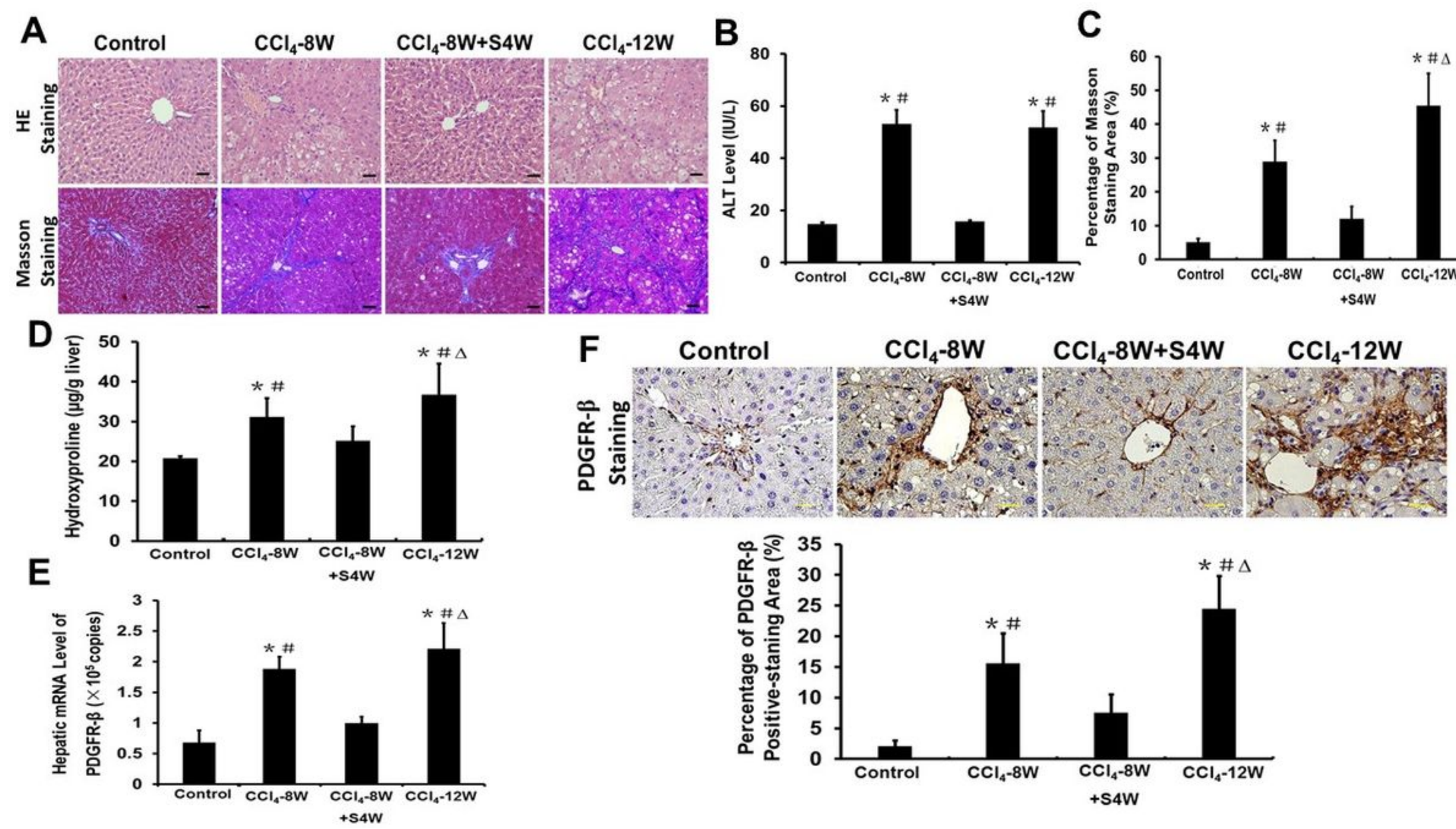

Figure 2

Expression of PDGFR- $\beta$ in fibrotic livers treated with $\mathrm{CCl} 4$. Liver fibrosis was induced in mice by treatment with $\mathrm{CCl} 4$ (CCl4 in olive oil, 1:9 (v/v), $2 \mathrm{ml} / \mathrm{kg}$ by intraperitoneal injection twice weekly) for 8 and 12 weeks 
(referred to as $\mathrm{CCl} 4-8 \mathrm{~W}$ and $\mathrm{CCl} 4-12 \mathrm{~W}$ ). Eight CCl4-8W mice were randomly selected to discontinue CCl4treatment for another 4 weeks for the spontaneous regression of fibrosis (referred to as CCl4-8W+S4W mice). Mice treated with the same dosage of olive oil for 12 weeks served as the control group. ( $n=8$ per group) (A) Representative micrographs of hepatic histology stained with H\&E (200x) and Masson (100x). Scale bars $=100 \mu \mathrm{m}$. (B) Comparison of serum ALT level in CCl4-treated and control mice. (C) Comparison of the Masson staining area in CCl4-treated and control groups. (D) Comparison of hydroxyproline content in liver tissue in CCl4-treated and control groups. (E) Comparison of hepatic PDGFR- $\beta$ mRNA levels in CCl4-treated and control groups. (F) Representative micrographs of hepatic PDGFR- $\beta$ expression stained by immunohistochemistry analysis (200x, Scale bars $=100 \mu \mathrm{m})$, and comparison of the percentage of the PDGFR- $\beta$ positive-staining (brown) area in CCl4-treated and control groups. In all panels, $₫ p<0.05$ versus control group; $₫ p<0.05$ versus $C C l 4-8 W+S 4 W$ group; $\triangle p<0.05$ versus CCl4-8W group.

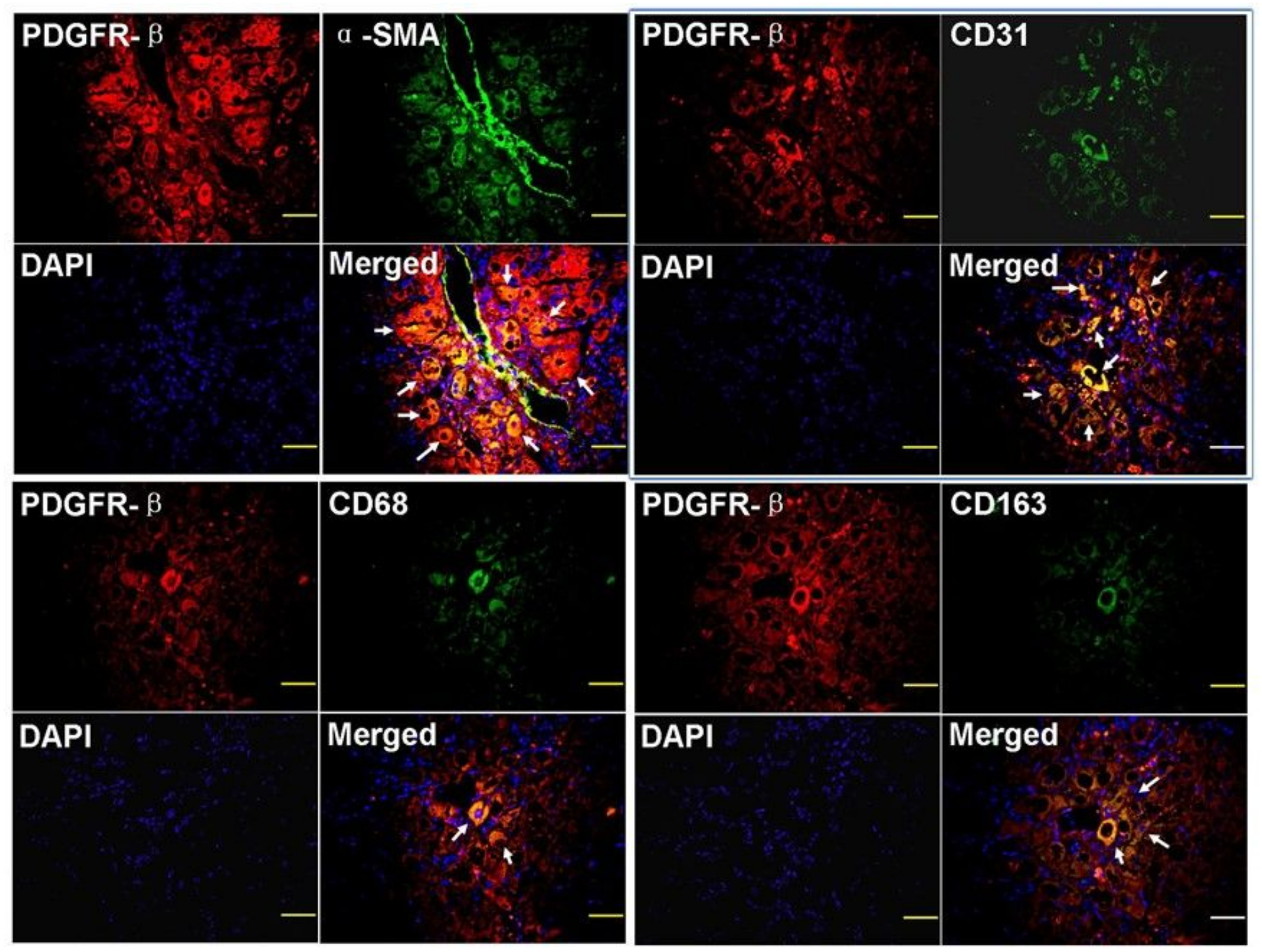

\section{Figure 3}

Immunofluorescent co-localization of PDGFR- $\beta$ and a-SMA, CD31, CD68 or CD163 in livers with advanced fibrosis. Representative fluorescent images of PDGFR- $\beta$ and a-SMA, CD31, CD68 and CD163 in liver 
sections from mice with CCl4-treatment for 12 weeks, which were separately stained with specific first antibodies and visualized by second antibodies, and counterstained with DAPI for nuclei staining. The merged images show the yellow color area by overlaying images of the counterstaining. Images were recorded at original magnification (400x). Scale bars $=100 \mu \mathrm{m}$.
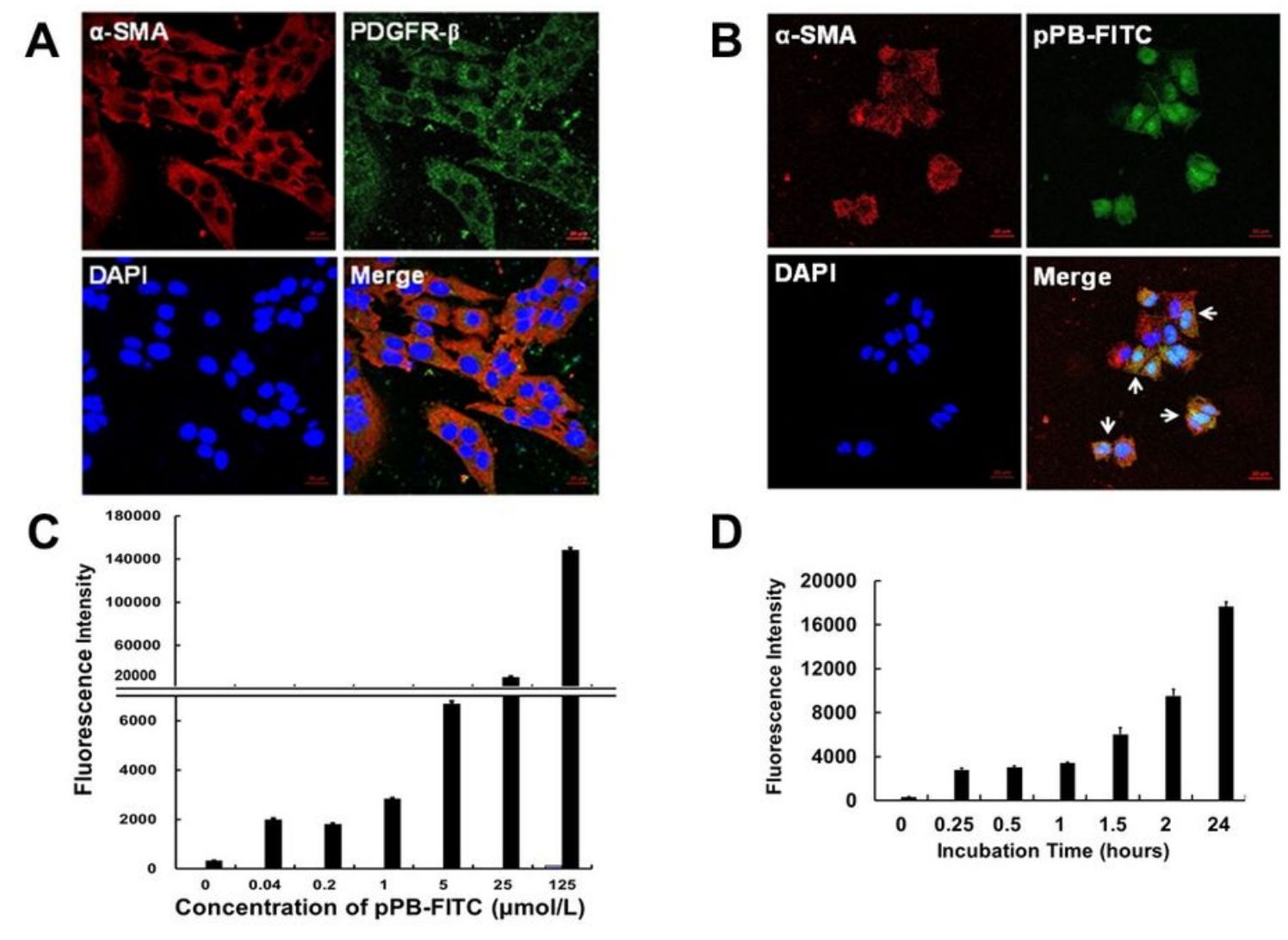

D

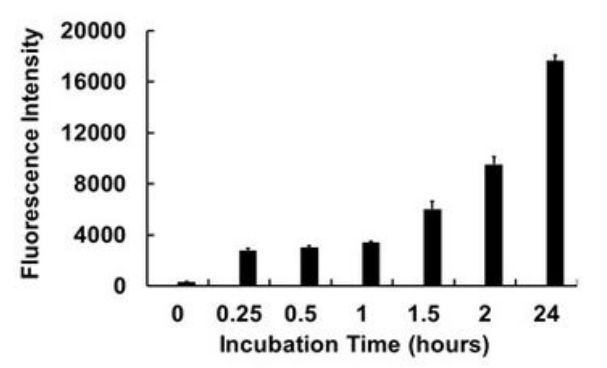

\section{Figure 4}

Binding characteristics of synthetic pPB cyclic peptides with cultured-activated human HSCs in vitro. Human HSC-LX2 were cultured for 24 hours after thawing and used for further experiments. (A) Representative fluorescent images of a-SMA and PDGFR- $\beta$ in HSC-LX2 stained with specific first antibodies and visualized by secondary antibodies, and counterstained with DAPI for nuclei staining. Images were recorded at original magnification (400x). Scale bars $=100 \mu \mathrm{m}$. (B) Representative fluorescent images of HSC-LX2 incubated with $1 \mu \mathrm{mol} / \mathrm{L}$ of FITC-labeled pPB (pPB-FITC) (green) for 24 hours at $37^{\circ} \mathrm{C}$ in dark, and counterstained with a-SMA antibody (red). The nuclei were stained with DAPI (blue), and the merged images show the yellow color areas pointed by white arrows. Images were recorded at original magnification (400x). Scale bars $=100 \mu \mathrm{m}$. (C) HSC-LX2 were incubated respectively with the pPB-FITC solution at concentrations of $0,0.04,0.2,1,5,25$ and $125 \mu \mathrm{mol} / \mathrm{L}$ for 1 hour at $37^{\circ} \mathrm{C}$ in dark. The change of the mean fluorescent intensity in HSC-LX2 analyzed with flow cytometry was shown. All experiments were undertaken in triplicates. (D) HSC-LX2 were incubated respectively with the pPBFITC solution $(1 \mu \mathrm{mol} / \mathrm{L})$ for $0,15,30,60,90,120$ minutes and 24 hours at $37^{\circ} \mathrm{C}$ in dark, and the change of the mean fluorescent intensity in HSC-LX2 analyzed with flow cytometry was shown. All experiments were undertaken in triplicates. 

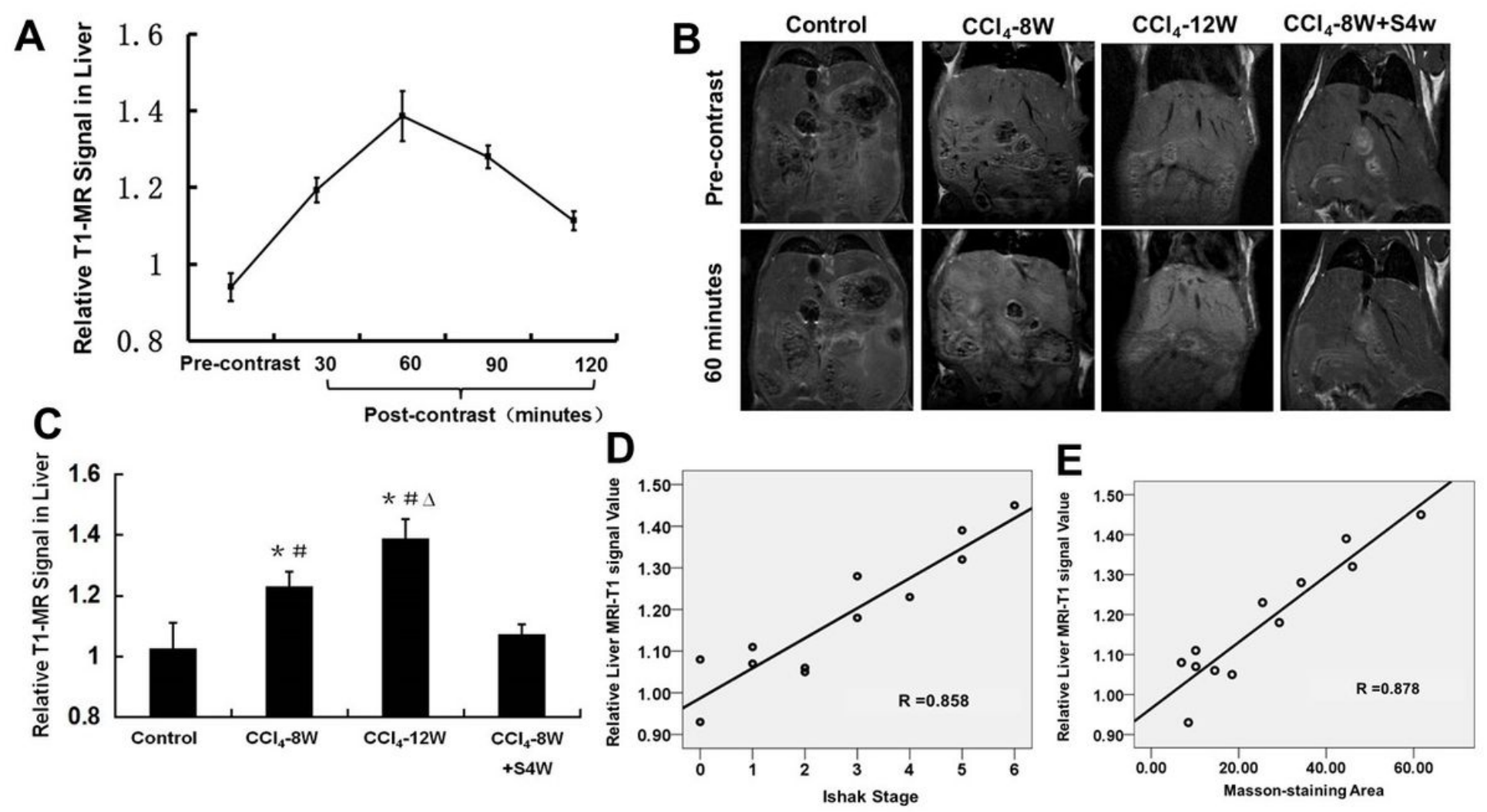

\section{Figure 5}

Imaging liver fibrosis using an MRI modality with pPB cyclic peptides labeled with Gd through DOTA (pPB-DOTA-Gd) as a tracer in mice. (A) Comparison of the relative liver T1-MR signal value in mice with CCl4-treatment for 12 weeks prior to and 30, 60, 90 and 120 min after pPB-DOTA-Gd injection $(n=3)$. (B) Representative hepatic T1-weighed MR images of the control and TAA-treated mice prior to and 60 min after pPB-DOTA-Gd injection. (C) Comparison of the relative liver T1-MR signal value in the control and TAA-treated mice 60 min after pPB-DOTA-Gd injection $(n=3)$. $『 p<0.05$ versus control group; $: p<0.05$ versus CCl4-8W+S4W group; $\triangle p<0.05$ versus CCl4-8W group. $(D, E)$ The correlation of relative liver T1MR signal value at 60 min post-injection of pPB-DOTA-Gd with Ishake stage (D) and Masson-staining area (E) was assessed. 